\title{
Timing of substorm related auroral oscillations
}

\author{
P. Martin ${ }^{1}$, N. E. Turner ${ }^{1}$, and J. Wanliss ${ }^{2}$ \\ ${ }^{1}$ Florida Institute of Technology \\ ${ }^{2}$ Presbyterian College
}

(Received October 26, 2007; Accepted August 16, 2008; Online published May 29, 2009)

\begin{abstract}
Previous studies have shown that auroral luminosity oscillations are often associated with substorms. Here we examine photometer data for the magnetospheric substorm on April 1, 2000 (expansive phase onset at 0525 UT) to study the detailed timing of the auroral oscillations relative to onset. Accurate timing information for the periodicities in the photometer data were determined using the wavelet transform. We find that the oscillations occur primarily during the recovery phase.
\end{abstract}

Key words: Aurora, substorm, wavelet, CANOPUS.

\section{Introduction}

Magnetospheric substorms are among the more consequential space weather effects, second in importance only to the massive and global space storms. Several studies have considered the effects of magnetohydrodynamic (MHD) waves on particle precipitation in the ionosphere (Berger, 1963; Davidson, 1990; Milan et al., 2001), and a strong link has been suggested between discrete auroral arcs and MHD waves (Samson et al., 1996; Wanliss and Rankin, 2002; Wanliss et al., 2002). MHD field line resonances (FLRs) are formed through wave coupling of compressional and shear Alfvén waves. The local compression of the magnetosphere at substorm expansive phase onset generates compressional MHD waves that encounter positive gradients in the Alfvén velocity leading to the excitation of shear Alfvén waves on the magnetic shells distant from the source of the onset. Because of the coupling of energy at spatially different locations, auroral activity is expected to occur at high and low latitudes. Theory predicts that the high latitude activity will feature a poleward phase shift of 180 degrees across the belt of auroral oscillation (Hughes, 1983; Liu et al., 1995). Similarly, the low latitude activity should exhibit a 180 degree equatorward phase shift and a temporarily varying parallel electric field will be established along the resonant field line (Block and Fälthammer, 1990; Liu et al., 1995). A consequence of this parallel electric field is the periodicities of the precipitating protons and electrons are anti-correlated. If there were no parallel electric field there should be no such phase difference and the precipitation of protons and electrons would likely be similarly modulated (Liu et al., 1995).

In this study we examine the timing of substorm associated auroral oscillations in order to determine which substorm phase they are associated with. The data are for the substorm on April 1, 2000 previously studied in detail by

Copyright (c) The Society of Geomagnetism and Earth, Planetary and Space Sciences (SGEPSS); The Seismological Society of Japan; The Volcanological Society of Japan; The Geodetic Society of Japan; The Japanese Society for Planetary Sciences; TERRAPUB.
Wanliss and Rankin (2002). They found $2.2 \mathrm{mHz}$ pulsations in magnetometer and photometer data. The proton and electron auroras were found to oscillate essentially out of phase, and the variation of phase across the peak in the luminosity resonance followed the pattern expected for the coupling of resonant Alfvén modes by normal compressional modes in the magnetotail. The previous work established accurate frequency information, but here we wish to establish detailed timing of the oscillations, a task for which the multiple filters of the wavelet transform are best suited.

\section{Data Description}

Wanliss and Rankin (2002) used photometer data from the Gillam meridian scanning photometer in northern Canada and found luminosity oscillations associated with magnetospheric substorms. They examined photometer and magnetometer data of a magnetospheric substorm on April 1, 2000 which had its expansive phase onset at 0525 UT. They examined the proton $(486.1 \mathrm{~nm})$ and electron $(557.7 \mathrm{~nm}, 630.0 \mathrm{~nm})$ auroras. The $486.1 \mathrm{~nm}(\mathrm{H} \beta)$ emission is caused by precipitation of protons of tens of $\mathrm{keV}, 557.7 \mathrm{~nm}$ from hot electrons of several $\mathrm{keV}$, and $630.0 \mathrm{~nm}$ from warm electrons of a few hundred $\mathrm{eV}$. This particular substorm occurred during a moderate space storm recovery phase with minimum $D_{\text {st }}=-60 \mathrm{nT}$. Figure 1 shows the $557.7 \mathrm{~nm}$ photometer data from the Gillam station (GILL). The data show brightness (Rayleighs) as a function of latitude and time. Each meridian scan takes one minute to complete, and data are organized into 17 latitudinal bins. The substorm growth phase is indicated by the steady equatorward motion of the auroras prior to $0525 \mathrm{UT}$, indicative of stretching of the inner magnetotail (Wanliss et al., 2000). At this time the emissions brighten and begin to move poleward, indicative of dipolarization of the previously stretched field lines.

A Fourier spectrogram of each wavelength revealed a peak around $2.2 \mathrm{mHz}$ (Wanliss and Rankin, 2002). The $630.0 \mathrm{~nm}$ wavelength also showed peaks at 2.9 and $3.8 \mathrm{mHz}$. Using CANOPUS magnetometer data the Pi2 


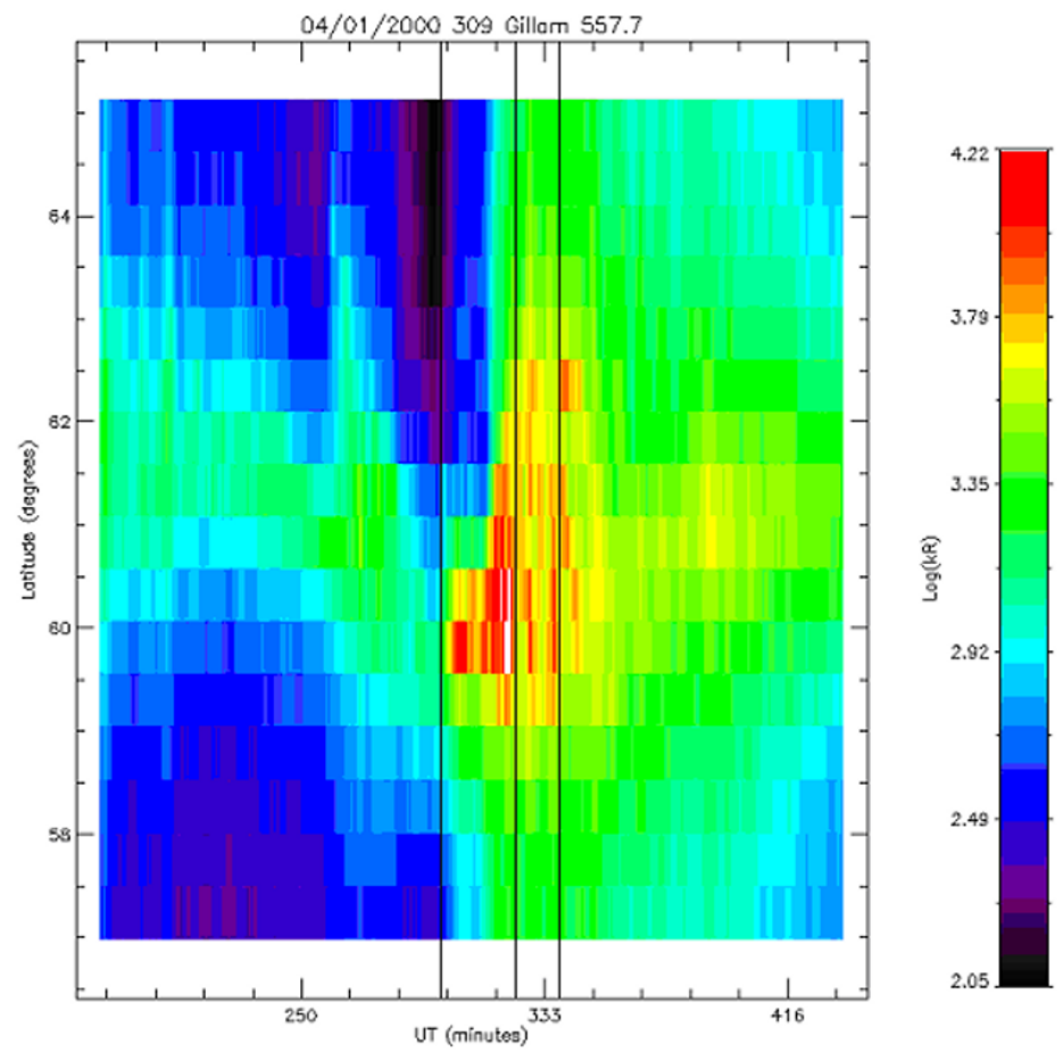

Fig. 1. Photometer data $(557.7 \mathrm{~nm})$ from the Gillam station for April 1, 2000 where substorm onset begins at the 309th minute of the day. The first vertical line indicates substorm onset and the two other lines indicate an equatorward intensification of the aurora.

pulsation was found to be localized between 67.4 and 69.7 degrees geomagnetic latitude. A phase correlation analysis was performed to determine if the spectral peaks might be related to field line resonances (FLR). The signals at 66.6, 66.1, and 65.6 degrees magnetic latitude, were anticorrelated which is consistent with a FLR and a parallel electric field. The phase shift around $2.2 \mathrm{mHz}$ showed a 120 degree phase shift at $\sim 66$ degrees for the $557.7 \mathrm{~nm}$ line which is lower than the theoretically required 180 degrees expected for a FLR (Chen and Hasegawa, 1974). For the $630.0 \mathrm{~nm}$ emission there was a 100 degree phase change which is qualitatively consistent but not quantitatively consistent with the FLR theory.

\section{Wavelet Analysis Method}

As with our previous work we produce a spectrogram of the photometer data for the substorm on April, 12000. Spectral analysis yields excellent frequency resolution for the peaks in the spectrogram, but poor timing information. In this study we seek good timing and frequency information, thus the wavelet transform is used to identify significant spectral power in frequency/time space. The varying size of the frequency/time bins makes it easier to identify wave power in time compared to the fixed size of the frequency/time bins in the windowed Fourier transform. Obviously, good time information results in decreased frequency resolution.

The output of the wavelet transform is both time and frequency dependent which is similar to the windowed Fourier transform. However, one obvious difference is the variable time length of the wavelet which results in a variable scale size or frequency band. The user does not need to know the frequency band to be searched for significant waves. The time span of the wavelet determines the frequency of the band in the spectrum. The wavelet makes multiple passes through the time series, adjusting its size for each pass. This is necessary to guarantee a complete period will fit in the wavelet and be recognized as a true wave, not a discontinuity. The resulting wave power is distributed in frequency and time. The highest possible frequency of the wavelet transform is the Nyquist frequency which is determined by the sampling rate. The lowest possible frequency is determined by the time length of the time series. The version of the wavelet transformed used in this study, required a minimum of eight complete wavelets to fit in the time series. The time length of the wavelet determines the lowest possible frequency. The multiple scale sizes also provide an advantage, in terms of timing issues, over the windowed Fourier transform. There is little power leakage along the time axis.

The power element of the wavelet transform represents the variance of the original time series at a specific range of time and frequency. Percival and Walden $(1993,2000)$ and Torrence and Compo (1998) showed the distribution of the power elements of the windowed Fourier and wavelet spectrums are similar to a chi-square distribution with 2 degrees of freedom. Therefore, analysis of variance (ANOVA) can be performed on the output to determine an accurate estimate of the frequency/time spectrum of the time series or the power spectral density (PSD). 


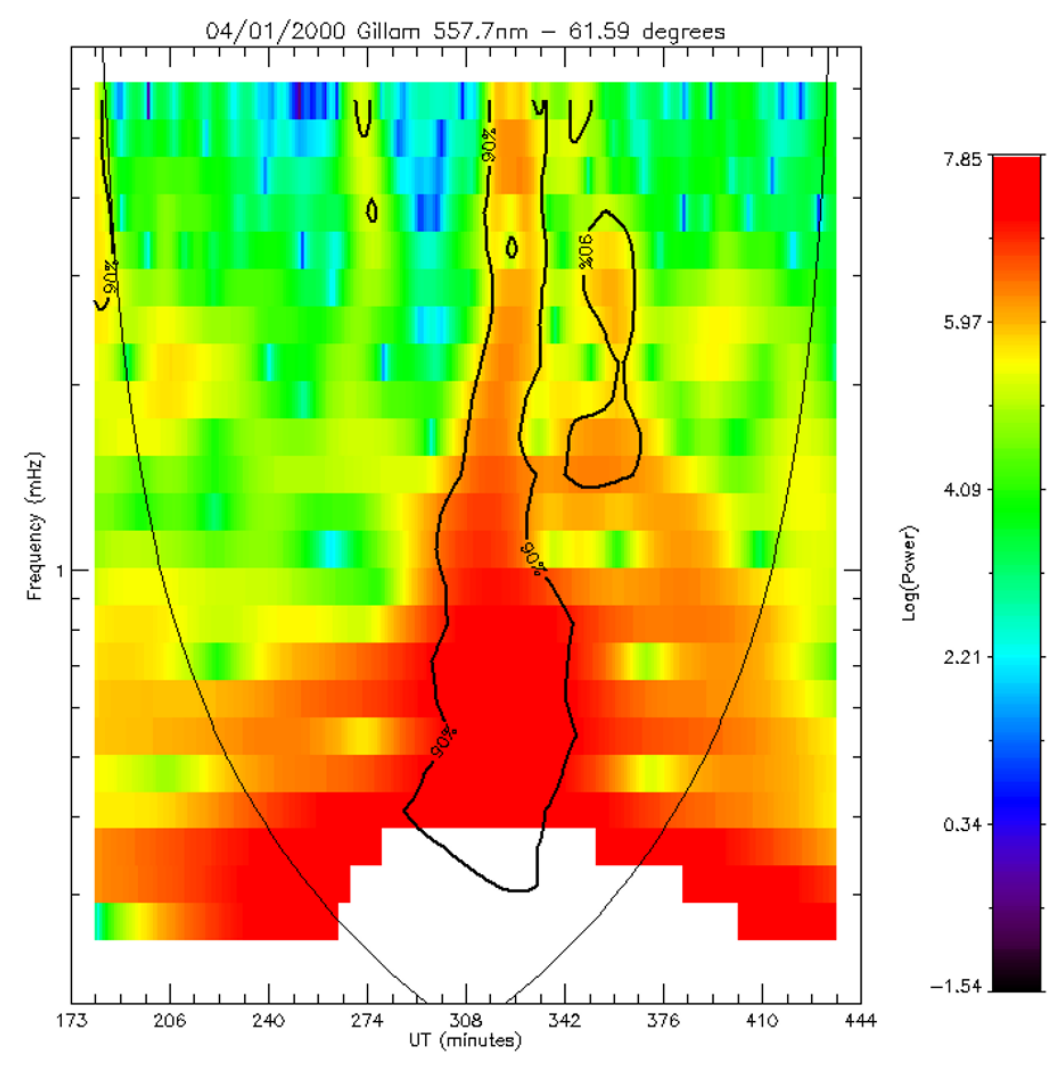

Fig. 2. The wavelet spectrum of the $557.7 \mathrm{~nm}$ photometer data measured at 61.6 degrees latitude. The regions outlined highlight significant spectral power.

$486.1 \mathrm{~nm}$
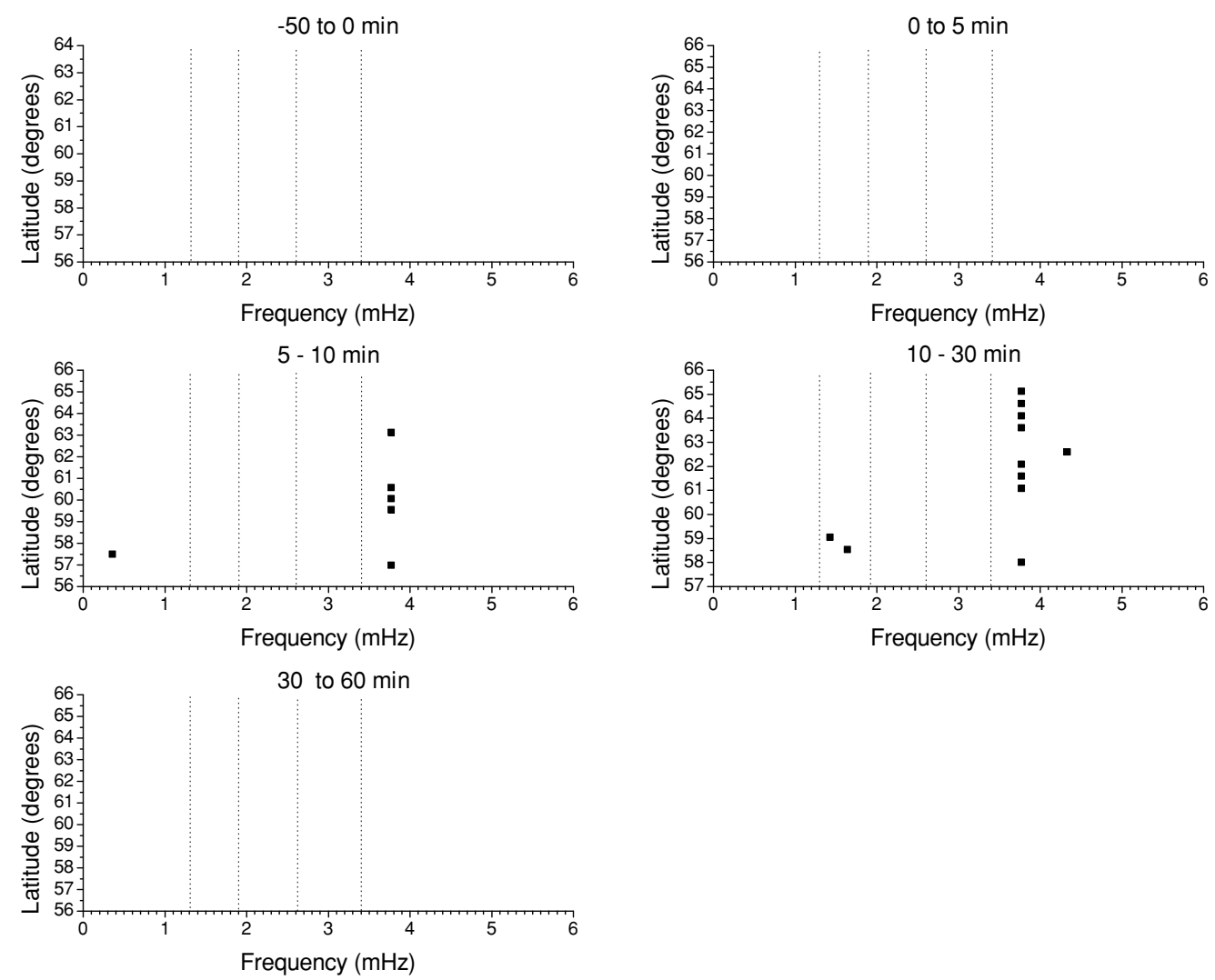

Fig. 3. The significant peaks in the $486.1 \mathrm{~nm}$ photometer spectra based on measurements at different latitudes. The data are divided into time intervals which represent the phases of the substorm. The vertical dashed lines mark the CMS frequencies greater than $1 \mathrm{mHz}$. 
$557.7 \mathrm{~nm}$
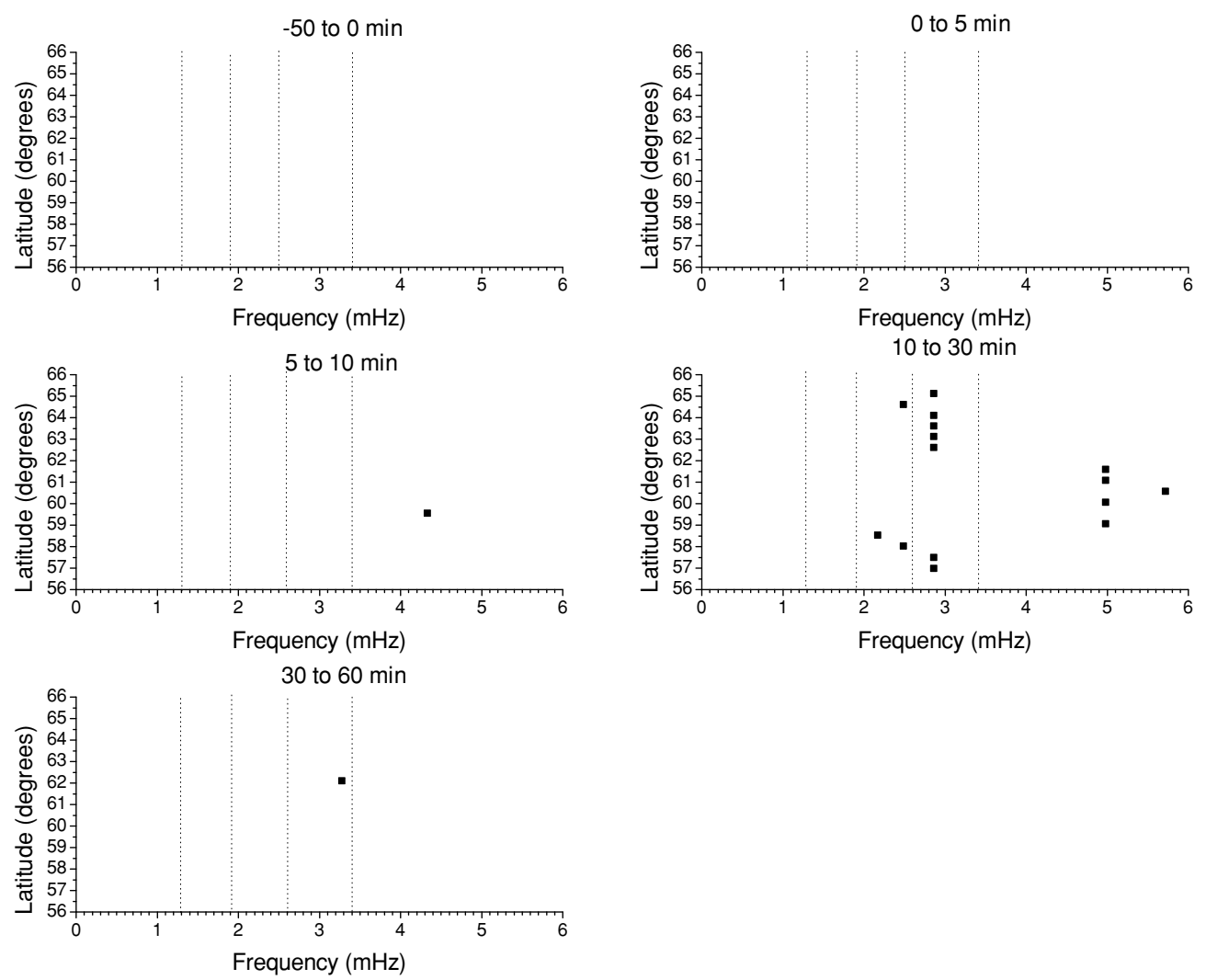

Fig. 4. The significant peaks in the $557.7 \mathrm{~nm}$ photometer spectra based on measurements at different latitudes.

\section{$630.0 \mathrm{~nm}$}
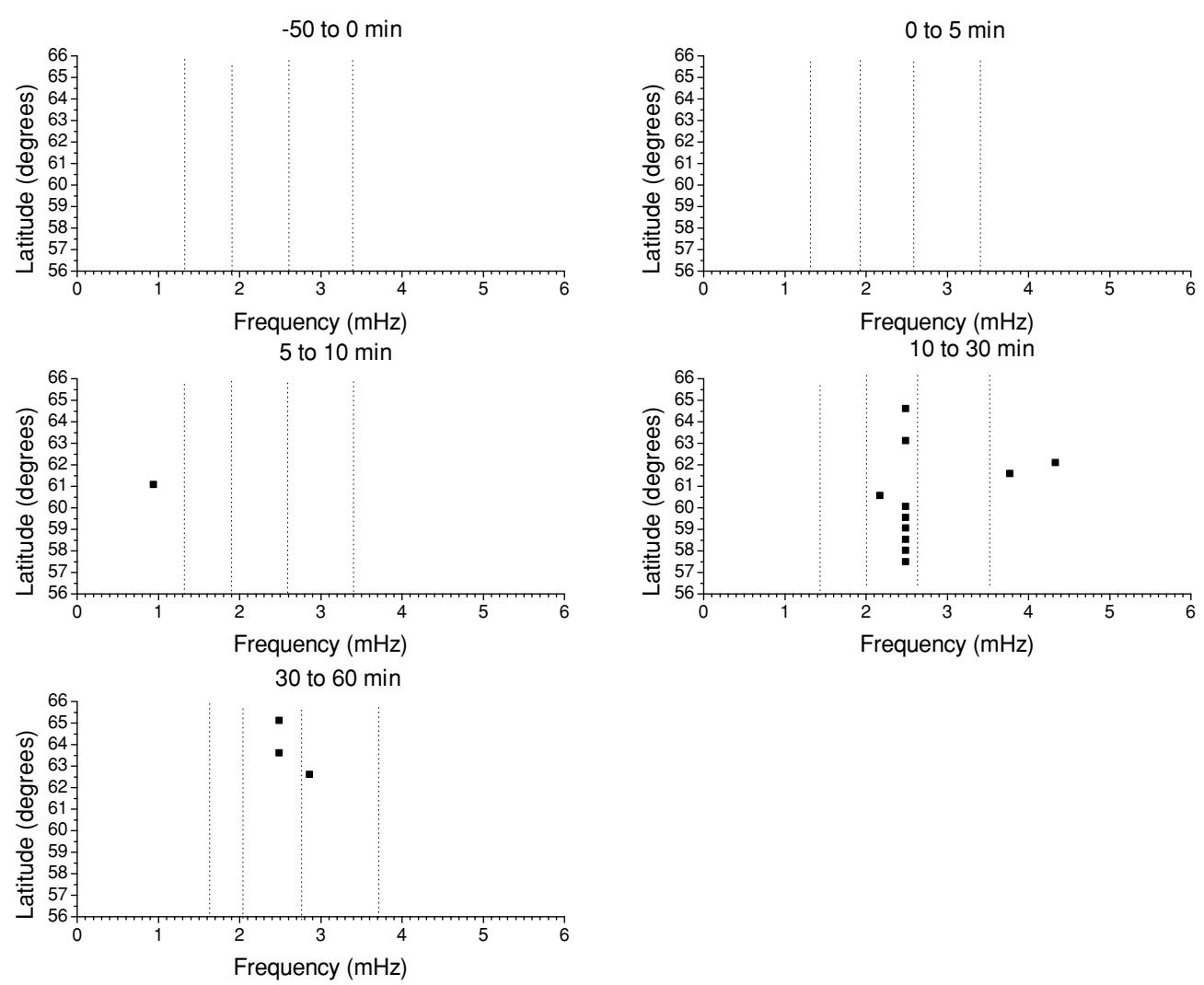

Fig. 5. The significant peaks in the $630.0 \mathrm{~nm}$ photometer spectra based on measurements at different latitudes. 
The power spectrum is determined from the baseline power level for each frequency band from which wave amplitudes can be measured (Percival and Walden, 1993, 2000; Torrence and Compo, 1998). The process of analysis of variance for the wavelet power spectrum assumes the time series has a mean power spectrum (Torrence and Compo, 1998). If a peak in the spectrum is significantly above the background then it can be assumed to be a true feature with a particular percent confidence (Torrence and Compo, 1998). A $90 \%$ confidence level was calculated for each scale which spans the entire length of the time series (256 minutes). Any random power variable with a value less than the $90 \%$ confidence level has a $90 \%$ probability of being the true mean and was considered to be part of the background. A power value greater than the confidence level is considered extraordinary and therefore significant. Any significant power value is considered to be a real peak in the spectral estimate.

We have used the complex valued Morlet wavelet over a 256 minute interval ( \pm 128 minutes from substorm onset). The time resolution of the continuous wavelet transform (CWT) increases at higher frequencies where the bandwidth gets larger (Press et al., 1992; Percival and Walden, 2000). For this analysis, the transform is set up for 56 scales or frequency bins; there are 5 subscales for every scale and there are 11 scales. These scales span $0.47 \mathrm{mHz}$ to $500 \mathrm{mHz}$ which is the Nyquist frequency. The first 18 scales $(0.47$ to $5.73 \mathrm{mHz}$ ) were used for this analysis. Figure 2 shows the spectrogram for the $557.7 \mathrm{~nm}$ data with the $90 \%$ confidence level indicated.

\section{Results}

The significant peaks from the seventeen latitudes for each of the three auroral wavelengths are combined to create Figs. 3 through 5. The spectra are summarized according to latitude and frequency across the entire 256 minute interval around the substorm onset which is marked as time equal to zero. The error bars indicate the bandwidth of the peaks. The error bar range marks high and low frequencies where the signal/noise drops below a value of 1 . The vertical dashed lines mark the CMS frequencies greater than $1 \mathrm{mHz}$ as identified by Samson et al. (1991) (1.3, 1.9, 2.7 and $3.3 \mathrm{mHz}$ ).

We find that spectral peaks in the 3 observed auroral wavelengths $(486.1,557.7$, and $630.0 \mathrm{~nm}$ ) have a NorthSouth alignment during the expansion phase of the substorm. The North-South arrangement is a result of the spectral peaks from the 17 different latitudes possessing the same frequency. In each of the three studied auroral wavelengths no significant wave activity is observed during the growth and early expansive phases of the substorm ( -50 to $0 \mathrm{~min}$, and 0 to $5 \mathrm{~min}$ ). Figures 3 through 5 show that the peaks in the spectrum form a North-South arrangement near one of the CMS frequencies, with most significant activity occurring from 10 to 30 minutes after substorm onset when the expansion phase is well developed.

\section{Discussion}

The study by Wanliss and Rankin (2002) showed how oscillations in the aurora were tied to substorm initiated, field line resonances. Here we have considered the timing of the FLR. Whereas the previous study accurately determined the frequency of the auroral oscillations, here we have utilized the wavelet transform to achieve accurate and quantitative isolation of the timing of significant oscillations. We find that the auroral oscillations for both electron and proton auroras do not begin at substorm expansion phase onset, but several minutes after the identified onset, and at a relatively localized latitude range. The oscillations intensify throughout the rest of the expansion phase across a wide range of latitudes, before dying down during the substorm recovery phase.

Acknowledgments. The Gillam meridian scanning photometer is owned and operated by the Canadian Space Agency. Work on this project by PM and NT was supported by NSF grant \#0454685.

\section{References}

Berger, S., Giant pulsations in the magnetic field and pulsating aurora, Planet. Space Sci., 11, 867, 1963.

Block, L. and C.-G. Fälthammar, The role of magnetic-field-aligned electric fields in auroral acceleration, J. Geophys. Res., 95(A5), 5877-5888, 1990.

Chen, L. and A. Hasegawa, A theory of long-period magnetic pulsations, 1, Steady excitation of field line resonances, J. Geophys. Res., 79, 1024, 1974.

Davidson, G. T., Pitch-angle diffusion and the origin of temporal and spatial structures in morningside aurorae, Space Sci. Rev., 53, 45, 1990.

Hughes, W., Waves in magnetospheric plasmas: Editorial, Geophys. Res. Lett., 10(8), 601-602, 1983.

Liu, W. W. et al., Theory and observation of auroral substorms: a magnetohydrodynamic approach, J. Geophys. Res., 100, 79, 1995.

Milan, S. E. et al., Auroral forms and the field-aligned current structure associated with field line resonances, J. Geophys. Res., 106, 25825, 2001.

Percival, D. B. and A. T. Walden, Spectral Analysis for Physical Applications, pp. 196-258, Cambridge University Press, 1993.

Percival, D. B. and A. T. Walden, Wavelet Methods for Time Series Analysis, pp. 56-145, Cambridge University Press, 2000.

Press, W. H., S. A. Teukolsky, W. T. Vettering, and B. P. Flannery, Numerical Recipes in C, pp. 496-608, Cambridge University Press, 1992.

Samson, J. C., R. A. Greenwald, J. M. Ruohoniemi, T. J. Hughes, and D. D. Wallis, Magnetometer and radar observations of magnetohydrodynamic cavity modes in the Earth's magnetosphere, Can. J. Phys., 69, 928,1991.

Samson, J. C. et al., Observations of field line resonances, auroral arcs, and auroral vortex structure, J. Geophys. Res., 101, 17373-17383, 1996.

Torrence, C. and G. P. Compo, A practical guide to wavelet analysis, Bull. Am. Meteor. Soc., 79(1), 61-78, 1998.

Wanliss, J. A. and R. Rankin, Auroral substorm dynamics and field line resonances, Earth Planets Space, 54, 927, 2002.

Wanliss, J. A., J. C. Samson, and E. Friedrich, On the use of photometer data to map dynamics of the magnetotail current sheet during substorm growth phase, J. Geophys. Res., 105, 27673-27684, 2000.

Wanliss, J. A., R. Rankin, J. C. Samson, and V. T. Tikhonchuk, Field line resonances in a stretched magnetotail: CANOPUS optical and magnetometer observations, J. Geophys. Res., 107(0), 10.1029/ 2001JA000257, 2002.

P. Martin (e-mail: pmartin@fit.edu), N. E. Turner, and J. Wanliss 